\title{
Study of the Aerodynamic Characteristics on the Computed Flowfield During Thrust Reversers Operation
}

\author{
S. Bi ${ }^{\dagger}$, J. Mao, X. Han, K. Cai and F. Wang \\ Jiangsu Province Key Laboratory of Aerospace Power System, Nanjing University of Aeronautics and \\ Astronautics, Nanjing 210016, China
}

$\dagger$ Corresponding Author Email: mjkpe@nuaa.edu.cn

(Received October 14, 2020; accepted March 30, 2021)

\begin{abstract}
With the increasing of the bypass ratio of modern aero-engines, the problem of wing-engine interference is more prominent, especially for the layout design of engine nacelle. The reverse thrust cascade is widely used in turbofan engines with high bypass ratio. In order to meet the requirements of the integrated analysis of airframe, wing, engine nacelle and hanger, the aerodynamic characteristics of aircraft/engine integration configuration with reverse cascade is numerically studied via CFD (Computational Fluid Dynamics) method. The streamline distribution, iso-surface of total temperature, vorticity distribution and total reverse thrust efficiency on different engine nacelle layouts are compared and analyzed in details. The results show that the lift coefficient of the wing decreases by $21.2 \%$ and $45.02 \%$ respectively as the engine moves forwards horizontally by $11 \% \mathrm{~L}$ and $21.2 \% \mathrm{~L}$. The lift coefficient of the wing decreases by $2.4 \%$ and $4.82 \%$ respectively as the engine moves subsidence by $-3.5 \% \mathrm{~L}$ and $3.5 \% \mathrm{~L}$. The influential region of the reverser airflow in the radial and circumferential direction without airframe interference is significantly larger than that in the case with aircraft/engine integration. The reverser flow is susceptible to be interfered by the adjacent fuselage and wing sections, and the development of reverse thrust flow is significantly limited. Compared to the baseline nacelle location, the reverse thrust performs badly as engine nacelle moves backward and lateral horizontally. The total reverse thrust efficiency decreases gradually as the engine nacelle moves forward horizontally.
\end{abstract}

Keywords: Thrust reverser; Propulsion system integration design; Aerodynamics performance; Nacelle layout; Numerical simulation.

\section{NOMENCLATURE}

$\begin{array}{ll}\mathrm{a} & \text { horizontal forward extension } \\ \mathrm{b} & \text { nacelle subsidence } \\ \mathrm{c} & \text { lateral movement } \\ C N P R & \text { internal bypass pressure ratio } \\ \mathrm{F}_{\text {core }} & \text { ideal thrust of internal bypass } \\ \mathrm{F}_{\text {fan }} & \text { ideal thrust of bypass } \\ \mathrm{F}_{\text {total }} & \text { total thrust } \\ F N P R & \text { bypass pressure ratio } \\ \mathrm{P}_{\text {in }}^{*} & \text { total pressure of cascade inlet } \\ \mathrm{P}_{\text {out }}^{*} & \text { total pressure of cascade outlet } \\ R_{g} & \text { gas constant } \\ \mathrm{S} & \text { blade thickness } \\ \mathrm{T}_{\text {core }}^{*} & \text { total temperature of internal bypass inlet } \\ \mathrm{T}_{\text {fan }}^{*} & \text { total temperature of bypass inlet } \\ \mathrm{U} & \text { blade width } \\ \mathrm{v} & \text { velocity }\end{array}$

$\begin{array}{ll}\mathrm{H} & \text { blade height } \\ \mathrm{i} & \text { the numbers of nacelle layouts } \\ \mathrm{k} & \text { specific heat ratio } \\ 1 & \text { engine axial length } \\ L & \text { blade chord } \\ \dot{m}_{\text {core }} & \text { Internal bypass mass flow rate } \\ \dot{m}_{\text {fan }} & \text { bypass mass flow rate } \\ \mathrm{v}_{\mathrm{y}} & \text { axial velocity } \\ X & \text { blade spacing } \\ \alpha & \text { inlet angle } \\ \beta & \text { exhaust angle } \\ \rho & \text { density } \\ \sigma & \text { total pressure recovery coefficient } \\ \varepsilon & \text { relative total pressure recovery coefficient } \\ \eta_{\text {rev }} & \text { total reverse thrust efficiency }\end{array}$




\section{INTRODUCTION}

High bypass ratio turbofan engine has the advantages of low fuel consumption and low noise in flight. Therefore, it is widely used in military and civil aircraft. Fang (2008) noted in addition to developing key technologies such as high-pressure compressor, low-pollution combustor, efficient multi-stage low-pressure turbine and noise reduction, high-bypass turbofan engine also attaches importance to technologies such as the design of reverse thrust device, low-resistance nacelle design and propulsion system/aircraft integration. The distance of take-off and landing slip greatly increases after using high bypass-ratio turbofan engines. There are many kinds of deceleration methods in aircraft sliding, and turbofan engines with high bypass ratio are usually designed with thrust reverser devices to shorten the sliding distance (Du et al. 2007; Sha and Xu 2007; Rao 2014). On the one hand, the outstanding advantages of the thrust reverser are that it can decelerate obviously under the conditions of wet ground and ice. On the other hand, the reverse thrust device is also applied to the quick return flight in case of emergency stop and unsuccessful takeoff and landing. Adopting reverse thrust device make the runway running distance of the aircraft reduce during landing and the utilization rate of the airport runway improve. The petal type, grab type, cascade thrust reverser type are the common forms of thrust reverser device, and the cascade thrust reverser type has gradually become the first choice for designing thrust reverser device, as mentioned by Yetter (1995) and Jin et al. (2004). In view of this, many research institutions and scholars have carried out a lot of research on the reverser characteristics of the thrust reverser device.

The aerodynamic performance of reverse thrust cascade affects the reverse thrust performance of the cascade thrust reverser device. And the distribution of the reverse thrust airflow in the engine flow field is influenced by geometric parameters such as the shape size and structure layout of the cascade. He and Liu (2010a), He and Liu (2010b), He and Liu (2012), Zhang and Wang (2010), Shan et al. (2010) and Wang et al. (2017) researched the reverse thrust characteristics of two-dimensional cascade structure, and considered the influence of cascade consistency, inlet flow angle and outlet flow angle on axial thrust efficiency by adopting numerical calculation methods. The axial thrust efficiency increases with the increase of cascade consistency in a certain range, but it does not fluctuate much with increase of cascade consistency continually. When the inlet flow angle and outlet flow angle increase within a certain range, the reverse thrust efficiency increases; and when increasing inlet flow and outlet flow angle continually, the reverse thrust efficiency changes slowly or even decreases. Among the researches, Zhang and Wang (2010) considered the choke leakage in the two-dimensional structural model, which was closer to the actual situation. In addition, some scholars studied the aerodynamic performance of thrust reverser ca.00scade through the experimental test and numerical simulation combination method. Wang et al. (2018) aimed at the gradual reverse thrust plane cascade in a certain type of reverse thrust device, it was found that the end-wall of the reverse thrust cascade presented three-dimensional unsteady and rotating flow characteristics by usage of numerical simulation, oil flow diagram and PIV flow field test. The process of reverse thrust flow development and evolution is similar to the flow process in turbine cascade. Wang et al. (2019) investigated the aerodynamic performance and flow field characteristic of thrust reverser cascade at different inlet Mach numbers for wide-body aircrafts by using experimental and numerical simulation methods.

The increase of bypass ratio of modern aero-engines results in the increase of nacelle diameter, inlet resistance, hood friction, weight and wing interference. CFD and wind tunnel tests were conducted in the development of civil aircraft in order to meet the requirements of integrated design and analysis of airframe/wing, engine and hanger. (see Rossow et al. 1994; Li et al. 2012) Dang et al. (2018) investigated the aircraft and engine threedimensional powered flow field on a tail-mounted civil aircraft. The results showed that the flow field at fan and core nozzle exit in installed state was affected by the installation and the difference of thrust characteristic of engine was mainly driven by compression-expansion-recompression process in installed and isolated configuration, which was affected by the installation of fuselage/wing/pylon. Therefore, some scholars carried out an integrated analysis of aerodynamic characteristics with reverse thrust device.

In addition to gaining the performance of reverse thrust, some problems of thrust reverser have attracted the attention of scholars. When thrust reverser device works, there may be some phenomenon such as pushing reverse airflow to pick up foreign matters on the ground, reverse thrust airflow re-inhaling, failure of control surface and vibration of airflow hitting the fuselage. De Andrade et al. (2006) studied the influence about precision of aircraft geometric modeling on the calculation results of the thrust reverser flow field in the process of aircraft landing and sliding with full 3D numerical simulation method, which considering the influence of such structures as wingtips, flaps, leading edge fins, landing gear and spoiler. Based on CFD technology, Trapp and Oliveira (2003) carried out numerical simulations on the thrust reverser device of EMB170 aircraft, and analyzed the influence of the uplift and buoyancy caused by the encounter of reverse thrust airflow on both sides under the fuselage on the aircraft.

On the basis of the above research, many scholars have been carried out in-depth studies on the reverse thrust airflow re-inhaling characteristics and intake stability of engine reverse airflow. Chen Chuck (2001), Chen et al. (2016), Chen (2014), Yi et al. (2014), Wang et al. (2016), Chen and Hu (2017), Wang et al. (2017) and Zhao (2019) studied the characteristics of the cascade thrust device of wing nacelle layout at different Mach numbers. Qian et al. (2011), Zuo and Qian (2007), Li et al. (2012) and Duan et al. (2016) carried out numerical simulations 
on the characteristics of cascade thrust reverser device of tail hanging layout at different Mach numbers. Then, the problems of airflow impacting, external inhalation and re-inhaling of reverse airflow are analyzed.

The above studies are all about the thrust reverser characteristics of a single engine nacelle layout. However, there are few studies about the effect of different engine nacelle layouts on the characteristics of reverse thrust, especially for a tailmounted aircraft. In this paper, a business regional tail-mounted jet equipped with 2 turbofan hybrid exhaust engines model is established which equips with reverse thrust cascades. A full threedimensional numerical simulation of the turbulence flow field in the environment of a single engine is calculated. Then basing on aircraft propulsion system integration considerations, the whole aircraft (including the engine and reverse thrust device) is considered. The purpose of this is, on the one hand, to obtain the interference characteristics of aircraft fuselage interference on reverse thrust airflow, and on the other, to obtain the flow field characteristics of the thrust reverser cascade at different engine nacelle layouts.

\section{NUMERICAL MODEL AND GRID}

\subsection{Calculation Model}

In this paper, a regional jet with thrust reverser is selected as the research objective. Fig.1 shows the geometrical model of the wing-body-tail-nacelle landing tail crane and the mode of engine. The nacelle is placed at the rear of the fuselage. The regional jet is powered by two-mounted turbofan engines with a thrust reverser. Fig. 2 shows a schematic diagram of cascade structure. The reverse thrust cascade model adopts the geometric parameters of cascade blade in reference Asbury and Yetter (2000). Table 1 shows the geometric structure parameters of cascade. The flow angle $(\alpha)$ of inlet is $60^{\circ}$ and the flow angle $(\beta)$ of outlet is $40^{\circ}$. The thickness of the blade (S) is $0.457 \mathrm{~mm}$, the chord of the blade $(\mathrm{L})$ is $65 \mathrm{~mm}$, and the radial vertical height of cascade is $8.636 \mathrm{~mm}$.

The design of different nacelle layout is realized by changing the chord installation position and vertical position of engine nacelle.

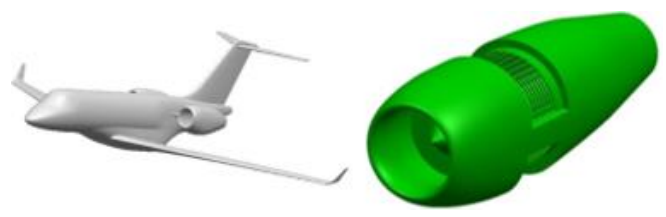

Fig. 1. Model of whole aircraft and engine.

Table 1 Cascade geometric structure parameters.

\begin{tabular}{|c|c|l|l|l|}
\hline$\alpha$ & $\beta$ & $S(\mathrm{~mm})$ & $L(\mathrm{~mm})$ & $H(\mathrm{~mm})$ \\
\hline $60^{\circ}$ & $40^{\circ}$ & 0.457 & 65 & 8.636 \\
\hline
\end{tabular}

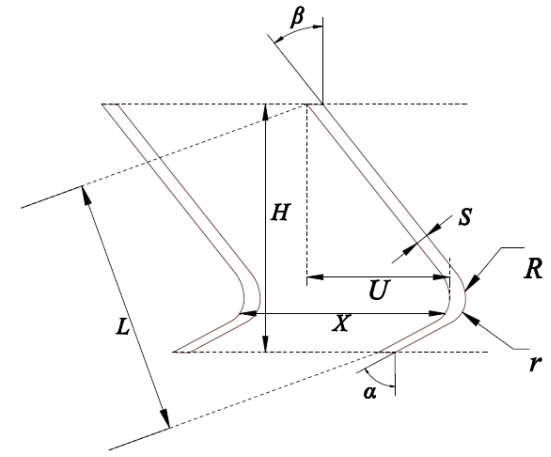

Fig. 2. Schematic diagram of cascade.

The nacelle course length is defined as $l$, the nacelle horizontal forward extension is defined as $a$, the nacelle subsidence is defined as $b$, the nacelle lateral movement is defined as $c$, the engine nacelle installation location selected in this paper has met the general layout requirements of the clearance between engine inlet lip and ground. Fig. 3 shows the schematic diagram of movement direction of engine nacelle, the model of forward extension $-1.15 \% \mathrm{a} / \mathrm{L}$ is named as case1, the basal model is named as case 2 , and the models of forward extension $11 \%$ and $22 \%$ are defined as case 3 and case 4 respectively. The model of lateral movement $1.15 \%$ of $c / L$ is defined case 5 , and the models of subsidence $-3.5 \%$ and $3.5 \% \mathrm{~b} / \mathrm{L}$ are defined as case 6 and case 7 respectively.

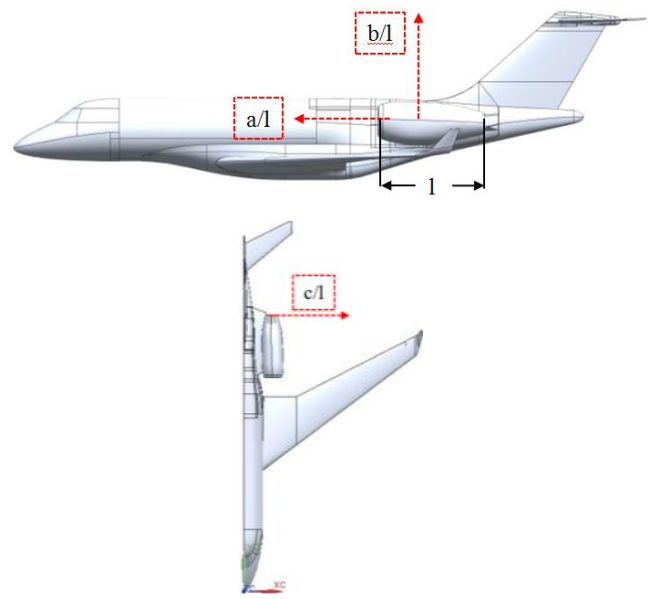

Fig. 3 Schematic diagram of movement direction of engine nacelle.

\subsection{Calculation Domain}

The numerical simulation method of aircraft/engine integrated reverser flow field is similar to that of a single engine. In order to get accurate results and describe the geometry of the plane as precisely as possible, the aircraft/engine integration model includes the fuselage, wings, vertical tail, horizontal tail, engine nacelles and hangers etc. Fig. 4 shows the calculation domain of the whole aircraft. The computational domain of numerical simulation of aircraft/engine integrated reverser flow field is a rectangular region. The plane is symmetrical, and the 
engines are mounted symmetrically on both wings. As a result, to save computing resources, the plane was split in two by an intermediate plane of symmetry. Only one side of the plane of symmetry is modeled and meshed.

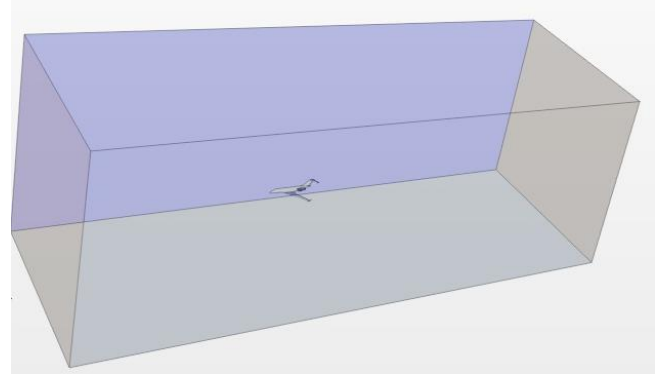

Fig. 4. Outflow computing domain.

After the plane's external disturbance flow computing domain is set up, the computing field of the engine flow needs to be set. When calculating the reverser turbulence flow field of the engine, the modeling process should be simplified appropriately. Thinking of the engine as a black box, and the flow inside of the engine is not simulated, only the details of flow field outside of the thrust reverser, inside of the engine inlet and the outside the engine are simulated. In addition, the calculation domain of a single engine is set as a rectangular region. The axial length of the calculation domain is about 10 times the engine length, the width and height of the calculation domain are 5 times engine length and 6 times engine length, respectively. The distance between the engine and the ground is the distance from the ground when the engine is actually installed.

\subsection{Calculation Grid}

The grid adopts polyhedral grid technology to divide the computing model, Fig. 5(a) shows plane surface grid, Fig. 5(b) shows local grid of engine nacelle and reverse thrust cascades. The characteristic size of the wall surface of cascade channel on the threedimensional reverse thrust cascade device is very small, and the grid density of cascade channel is relatively large. However, the outflow field of the reverse flow is larger, considering comprehensively the computational accuracy and resources, the mesh of cascade surface part is encrypted. The minimum size of polyhedral mesh is controlled at $0.001 \mathrm{~m}$, and the boundary layer grid is constructed among the core parts of the thrust reverser device, such as the wall surface of cascade channel, inlet wall and bypass channel. A prismatic grid generator is used for the boundary layer, the thickness of the first layer is $0.00001 \mathrm{~m}$, and the growth rate of the surface grid is 1.3. The mean of $y+$ is less than 3 , which satisfies the requirement of the turbulence model used in the calculation. The total number of grids in the numerical simulation of the flow field of singleengine reverser flow is about 12.2 million. After the grid independence test, as shown in the Fig. 6, the reverse thrust efficiency is basically unchanged when the grid size is 18.75 million, which meets the calculation needs.

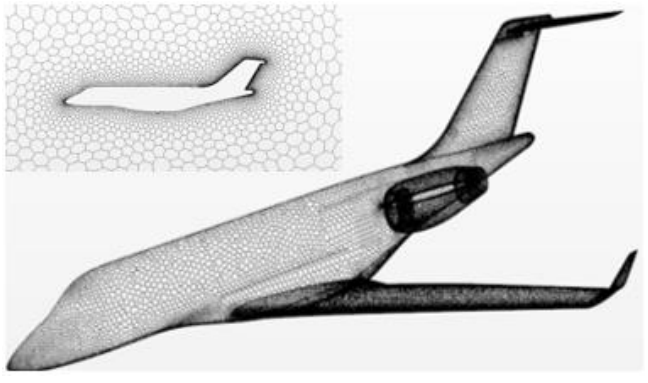

(a) plane surface grid

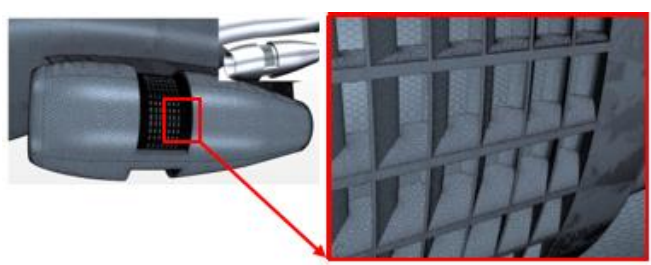

(b) engine nacelle gird

Fig. 5. Grid of calculation.

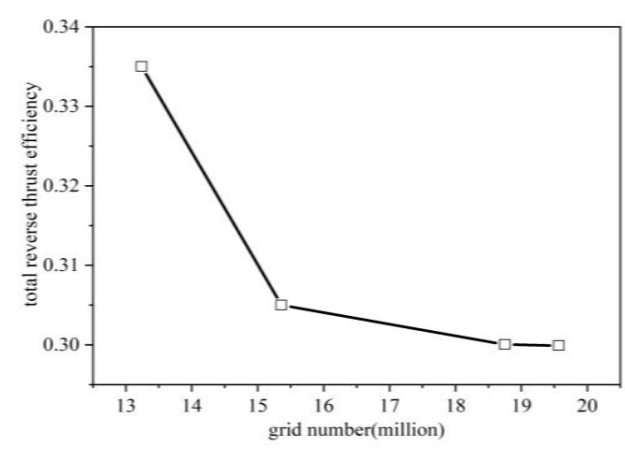

Fig. 6. Grid independence test.

\section{NUMERICAL METHOD AND SET UP}

\subsection{Numerical Method}

In order to verify the accuracy of numerical model and choose an appropriate turbulence model, the DLR-F4 wing-body assembly model is modelled with three turbulence models. The numerical verification of aircraft outflow turbulence was performed by comparing with the publicly available aircraft wind tunnel test data (Levy et al. 2002). Fig. 7 shows the DLR-F4 wing-body assembly model for resistance prediction meeting. The mean of $y+$ is near 1 , which satisfies the requirement of the turbulence model used in the calculation. The N-S equation based on Reynolds mean is used in the calculation, the inlet Mach number is 0.75 , and the angle of attack was 0.49. Spalart-Allmaras (SA), $\mathrm{V}^{2}$ $\mathrm{f}$ (V2F) and shear stress transport (SST) $k-\omega$ turbulence models are used for calculation respectively. The $k-\omega$ SST turbulence model is the best match with the experimental data, as shown in Fig. 8. The effects of low Reynolds number, compressibility and shear flow diffusion were considered in the $k-\omega$ SST turbulence model, which is suitable for flow calculation limited by wall surface. The turbulence model is used in this situation to calculate the flow characteristics of 
reverse thrust airflow, which is limited by the wall in the cascade passage. Therefore, the $k$ - $\omega$ SST turbulence model is used in the following calculations.

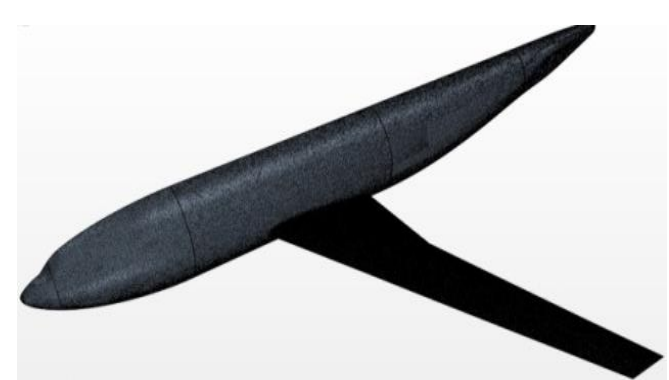

Fig. 7. DLR-F4 Wing-body assembly model.

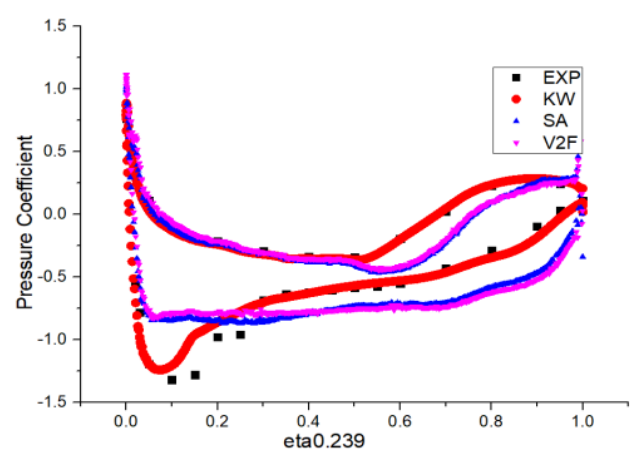

Fig. 8. Wing pressure coefficient distribution at the position of 0.239 spanwise station.

In order to simulate the applicability of turbulence models in the flow situation of reverse thrust, the simulation results of jet flow in crossflow were compared with the experimental results. For a detailed description of the experiment facilities Andreopoulos and Rodi (1984), Su and Mungal (2004). Fig. 9 shows the model of jet in crossflow. The cross flow passed through a channel, and both the length of the side of the cross section were $108 \mathrm{~mm}$, and the length of channel was $628 \mathrm{~mm}$. The jet flowed through a $250 \mathrm{~mm}$ round tube, and the diameter (D) of tube was $8 \mathrm{~mm}$.

The numerical calculation uses the same boundary conditions as the experiment. The bulk velocity of crossflow inlet is $9.08 \mathrm{~m} / \mathrm{s}$, the turbulence intensity is $1.5 \%$ and the $\operatorname{Re}$ is $6.24 \times 104$. The maximum velocity of crossflow was located at a plane $1.5 \mathrm{D}$ upstream of the tube center, which was defined as $U_{\text {cross. }}$ The bulk velocity of jet inlet was $37.72 \mathrm{~m} / \mathrm{s}$, the turbulence intensity is $7 \%$ and the Re is $1.92 \times$ $10^{4}$. All the wall surfaces of the grid meet the requirement that $y+$ is around 1 . The influence of boundary layer on jet flow can be better simulated.

Fig. 10 shows the comparison of calculation results and experimental results under different turbulence models. The N-S equation based on Reynolds mean is used in the calculation. SA, V2F and shear stress transport (SST) k- $\omega$ turbulence models are used for calculation respectively. Fig. 10(a) shows that three turbulence models could well predict overall variation trend of velocity along the flow direction at the location $(\mathrm{z} / \mathrm{D}=1.5)$, but the SST $\mathrm{k}-\omega$ turbulence model performance better at the location of about $x / D=1.5$. Fig. $10(b)$ shows that the three turbulent model show similar results to the experiment data at the plane $(x / D=0)$, and the SST $k-\omega$ turbulence model obtains a result closer to the experimental value between the region $(\mathrm{x} / \mathrm{D}=0.5)$ and the region $(\mathrm{x} / \mathrm{D}=1.5)$

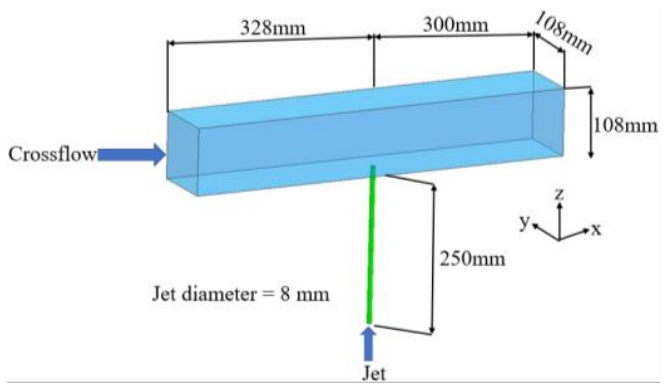

Fig. 9. Model of jet in crossflow.

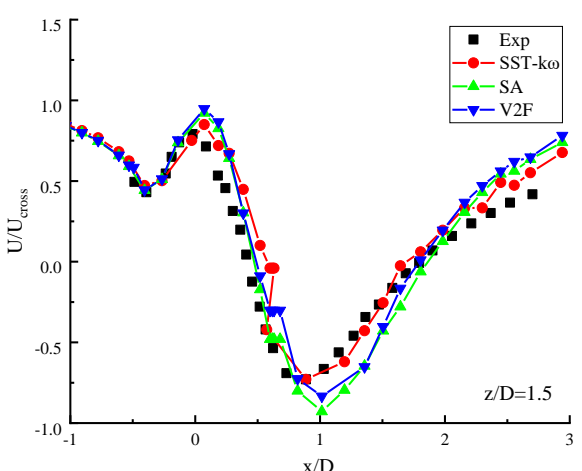

(a)

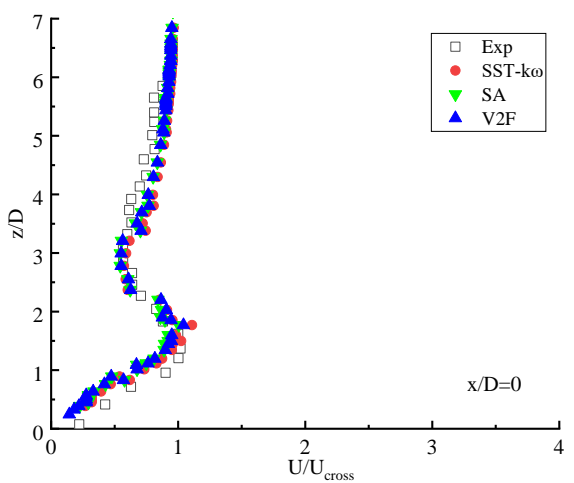

(b)

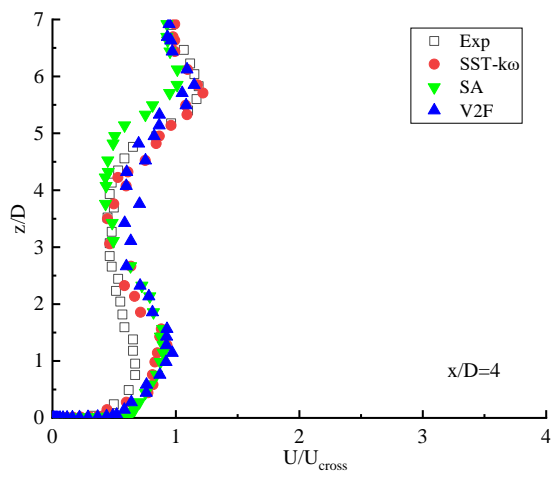

(c)

Fig. 10. Velocity component $U / U_{\text {cross }}$ at the plane $(\mathrm{y}=0)$. (a) $\mathrm{z} / \mathrm{D}=1.5$; (b) $\mathrm{x} / \mathrm{D}=0$; (c) $\mathrm{x} / \mathrm{D}=4$. 


\subsection{Computing Setup}

Fig. 10(c) shows that SST k- $\omega$ turbulence model obtains a better predictive value of velocity distribution along $\mathrm{z}$ direction at the plane $(\mathrm{x} / \mathrm{D}=4)$. So the SST k- $\omega$ turbulence model could well predict the situation of jet in crossflow, which is similar to case of reverse flow into the ambient flow.

Numerical simulation was carried out to study the turbulence field of the reverse airflow with different nacelle layouts at the sliding speed of $0.1 \mathrm{Ma}$, and ambient temperature is $288.15 \mathrm{~K}$, ambient pressure is 101325Pa. The Reynolds mean N-S equation was adopted and the turbulence model was $k-\omega$ SST model. The higher order numerical scheme is used to solve the equation, and the convergence criteria for residuals are $10^{-5}$. The flow conditions of the compressor, combustion chamber and turbine parts inside the engine are neglected in the modeling. Numerical simulation of engine inlet and exhaust and reverse thrust flow field are carried out merely. The inlet boundary of the outflow calculation domain is defined as the free flow boundary, which is the relative incoming flow Mach number. That is the landing slip speed of the aircraft, and the below boundary is defined as the solid wall.

Fig. 11 shows the diagram of engine inlet and exhaust calculation boundary conditions setting. The fan inlet face of the engine is given the pressure outlet boundary. The section of nozzle inlet is the exit section of internal bypass, and the inlet boundary of reverse thrust airflow is the inlet of external bypass. And the inlet boundary of the nozzle and reverse thrust airflow are defined as the inlet boundary of mass flow, which are given the corresponding working state of the air flow and temperature.
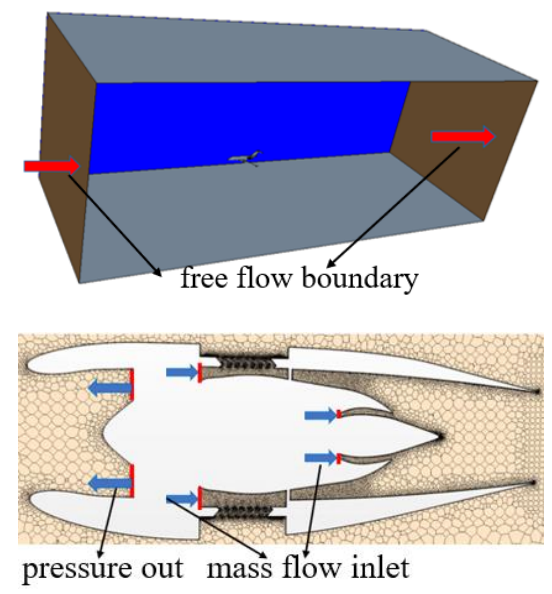

Fig. 11. Diagram of engine inlet and exhaust calculation boundary.

\section{THEORETICAL BASIS}

The turbofan engine of separate exhaust is that air flow of internal and external bypass respectively outflow from engine internal and external bypass. According to the ideal gas adiabatic state, the engine thrust of internal bypass and engine thrust of external bypass can be calculated by formula (1) and formula (2). Engine thrust of internal and external bypass together make up the total thrust of the engine, such as formula (3).

$$
\begin{aligned}
& \mathrm{F}_{\text {core }}=\dot{m}_{\text {core }} \times \sqrt{2 \frac{k R_{g} T_{\text {core }}^{*}}{k-1}\left[1-\left(\frac{1}{C N P R}\right)^{\frac{k-1}{k}}\right]} \\
& \mathrm{F}_{\text {fan }}=\dot{m}_{\text {fan }} \times \sqrt{2 \frac{k R_{g} T_{\text {fan }}^{*}}{k-1}\left[1-\left(\frac{1}{F N P R}\right)^{\frac{k-1}{k}}\right]} \\
& F_{\text {total }}=F_{\text {core }}+F_{\text {fan }}
\end{aligned}
$$

When the thrust reverser is turned on, the airflow from cross section of 1-1 is reversed at the cross section of $2-2$, as the Fig. 12 shown. The axial thrust of the cross section of 2-2 can be calculated by formula (4). The axial reverse thrust produced by cascade reverse thrust cascade can be calculated after postprocessing the results of flow field. The axial velocity is integrated with the flow rate by a plane at the exit of the cascade, the face of the integral is listed in the red box line at the Fig. 13. After calculating the axial force, the total reverse thrust coefficient can be calculated by the formula (5).

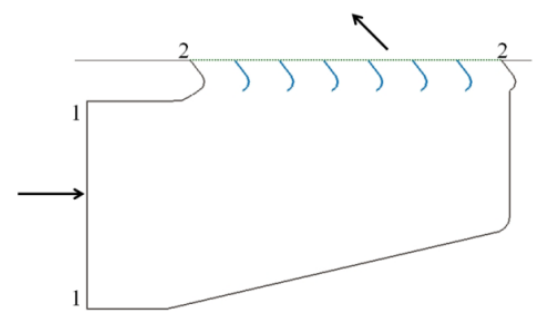

Fig. 12. Axial force of reverse thrust state.

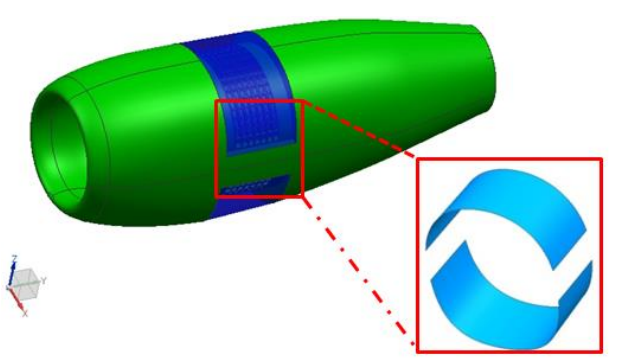

Fig. 13. Integral surface of cascade exit.

$$
\begin{aligned}
& F_{\text {axial }}=\int \rho V V_{y} d A \\
& \eta_{\text {rev }}=F_{\text {axial }} / F_{\text {total }}
\end{aligned}
$$

In the actual flow process, the expansion process is not isentropic due to viscous dissipation and other reasons, and there will always be total pressure loss when the gas flows through the cascade. The actual flow velocity at the outlet section of the cascade is less than the ideal flow velocity. At the same time, after the engine nacelle moves, the relative distance between the engine nacelle and the fuselage and wing changes, which will affect the outflow of 
reverse thrust airflow. In view of this, the total pressure recovery coefficient $\sigma$ is adopted in this study to measure the total pressure loss of airflow flow, and the total recovery coefficient is defined as formula (6). The total pressure recovery coefficients of different nacelle layouts is defined as $\sigma_{i}$ and the value of $i$ refers to the nacelle position. The relative total pressure recovery coefficient is defined as $\varepsilon$.

$$
\sigma=\frac{P_{\text {out }}^{*}}{P_{\text {in }}^{*}}=\frac{\frac{1}{m_{\text {out }}} \int P_{\text {out }}^{*} d m_{\text {out }}}{\frac{1}{m_{\text {in }}} \int P_{\text {in }}^{*} d m_{\text {in }}}
$$

$\varepsilon=\sigma_{\mathrm{i}} / \sigma_{2}(\mathrm{i}=1,2 \ldots 7)$

The lift of airplane is calculated from the result of numerical calculations, which is defined as $L$. The lift coefficient of airplane is defined as $C_{L}$ :

$$
C_{L}=L / \frac{1}{2} \rho \mathrm{v}^{2} S
$$

In the formula (8), $\rho$ is the density of the mainstream, and $v$ is the velocity of the mainstream. $S$ is the reference area, generally, the area of an airplane wing is selected.

The value of basal engine nacelle position $i$ is defined as 2 . The forward extension of a/L are $1.15 \%, 11 \%, 22 \%$, the corresponding value are case 1 , case 3 and case 4 . The lateral movement of $\mathrm{c} / \mathrm{L}$ is $1.15 \%$ the corresponding value of $i$ is defined 5 , and the subsidence of $\mathrm{b} / \mathrm{L}$ is $-3.5 \%$ and $3.5 \%$, the corresponding value of $i$ are defined as 6 and 7 . The dimensionless number $\varepsilon$ is used to compare with the change of reverse airflow flow loss at different engine nacelle layouts.

The calculation of reverse turbulence flow field in the engine installation state requires the overall modeling and grid division of aircraft, engine and thrust reverser, which requires large computational resources. In order to save computational resources, a series of numerical calculations of turbulence field of reverser thrust flow are carried out in three steps in this paper. Firstly, the effect of different engine layout on the aerodynamics of the aircraft is compared and analyzed. The second, several groups of 3D reverser flow field calculations are carried out in reverse thrust state of a single engine. The reverser flow details are captured, and the flow parameter distribution on the outlet section of the reverse thrust device is obtained. Then, the numerical simulation of reverser turbulence flow field of aircraft/engine integration is carried out to analyze the interference effect of aircraft body on the reverser flow field of engine.

\section{RESULTS AND DISCUSSION}

\subsection{Flight Performance at Different Engine Layouts}

Different nacelle layouts affect the aerodynamics of the aircraft and thus the flight performance of the aircraft. Cruising performance is an important parameter in aircraft design. Therefore, the pressure coefficients of different engine nacelle layouts under high altitude cruise condition are compared before calculating the aircraft/engine integration model with thrust reverser. Fig. 14 shows the pressure coefficient of the wing at different engine layouts. Fig. 14(a) gives the surface pressure coefficients of wing at three different horizontal positions of engine nacelle. When the engine nacelle moves forwards horizontally by $11 \% L$, the surface pressure coefficient on the upper side of wing increases obviously from 0.2 to 0.4 chord. When the engine nacelle continues to move forward by $22 \% L$, the distance between inlet of engine and wing reduces, and the effect of engine entrance on the flow near the wing become larger. The pressure coefficients distributions on upper side of the wing from 0.1 to 0.4 chord increases. The lift coefficient of the wing decreases by $21.2 \%$ and $45.02 \%$ respectively as the engine moves forwards horizontally by $11 \% L$ and $21.2 \%$ L. Fig12(b) gives the surface pressure coefficients of wing at three different nacelle layouts. The surface pressure coefficients of wing changes less as the engine nacelle moves along the vertical direction. The lift coefficient of the wing decreases by $2.4 \%$ and $4.82 \%$ respectively as the engine moves subsidence by $-3.5 \% \mathrm{~L}$ and $3.5 \% \mathrm{~L}$.

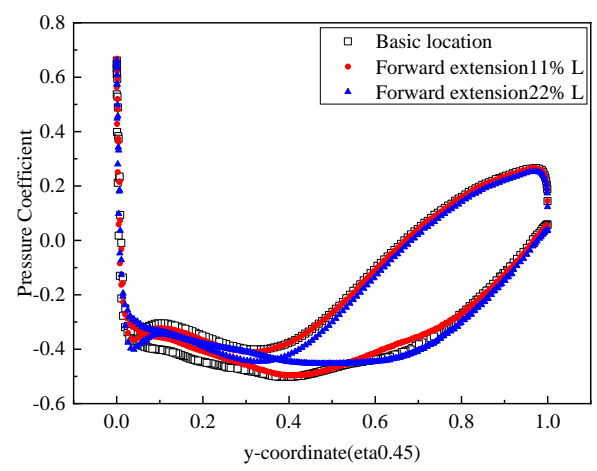

(a) horizontal direction

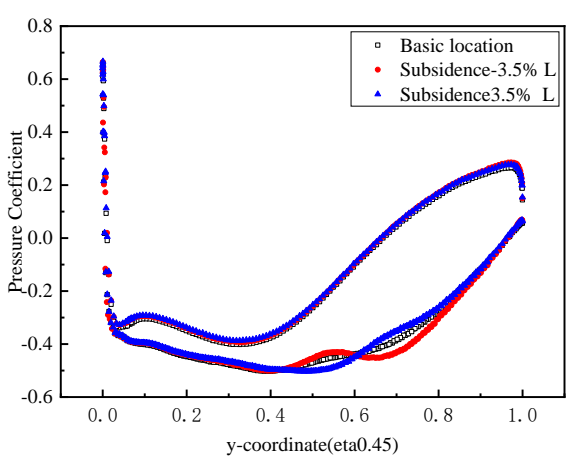

(b)vertical direction

Fig. 14. Wing surface pressure coefficients distributions at different engine layouts.

\subsection{Influence of Airframe Interference}

As mentioned above, different engine nacelle layouts will change aerodynamics parameters of aircraft. 


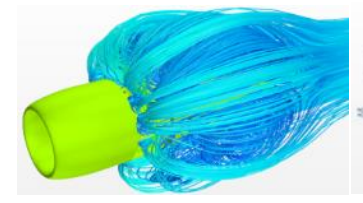

(a) $0.1 \mathrm{Ma}$

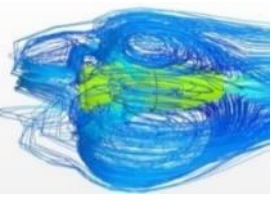

(b) $0.05 \mathrm{Ma}$
Fig. 15. Space streamline distribution.

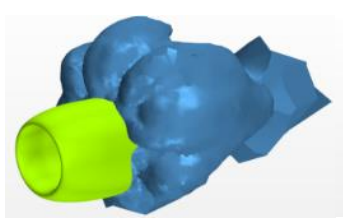

(a) $0.1 \mathrm{Ma}$

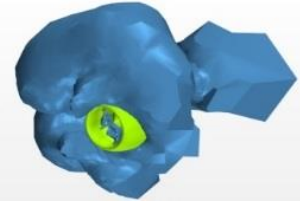

(b) $0.05 \mathrm{Ma}$
Fig. 16. Iso-surface of total temperature at $300 \mathrm{~K}$.

Therefore, the influence of fuselage interference on the thrust characteristics is analyzed firstly, and then the coupling effect between engine nacelles layout and thrust characteristics is analyzed. Fig. 15 shows the reverser flow streamline distribution of a single engine, the aircraft's relative Mach number are 0.1 and 0.05 respectively. Fig. 15 shows that after reverser flow ejecting, it firstly moves towards the front of engine, and then the reverser flow also eventually bends downstream. Fig. 15 shows that with the decrease of inlet Mach number, the obstruction of front inlet to reverser flow decreases and the radial range affected by reverser flow gradually increases, additionally the moving distance of reverser flow towards engine inlet increases.

Fig. 16(a) and Fig. 16 (b) show total temperature isosurfaces of engine at different running speeds, which is higher than the engine's surrounding environment of $288.15 \mathrm{~K}$. Therefore, the iso-surface with a total temperature of $300 \mathrm{~K}$ can reflect the influence range of reverser flow. When the incoming flow velocity decreases to $0.05 \mathrm{Ma}$, the reverser flow with a total temperature of $300 \mathrm{~K}$ has covered the area around the engine inlet section. Reverser flow occupies the space in front of the engine lip, which increases the influence range.

Fig. 17 shows reverser flow streamline distribution for different skidding speeds in the aircraft/engine integration calculation. With the decrease of the aircraft skidding speed, the radial influence range of the reverse airflow gradually increases. When aircraft skidding velocity is $0.1 \mathrm{Ma}$, the reverser flow is enhanced by the blocking effect of the incoming flow. The reverser flow flows to the back of the turning point, and there is no phenomenon of repeated inhalation. When the running speed decreases to $0.05 \mathrm{Ma}$, the momentum of the incoming flow decreases. Mixing loss between reverser flow and the inflow mainstream decreases, and the distance of reverser flow forwards movement increases. The turning point in the direction of reverser flow moves forward. According to Fig. 17(b), the reverser flow jet is inclined to the front of the engine. Due to the low momentum of the incoming flow, the reverser flow sprays out with a relatively large momentum after further acceleration in the reverse thrust cascade passage. Some reverser flow forms a vortex zone in front of the engine lip and its influence range increases. At the same time, it happens to a phenomenon of repeated inhalation.

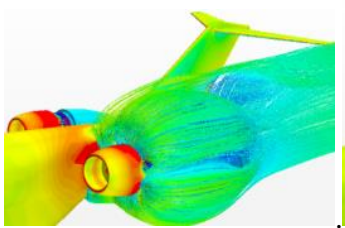

(a) $0.1 \mathrm{Ma}$

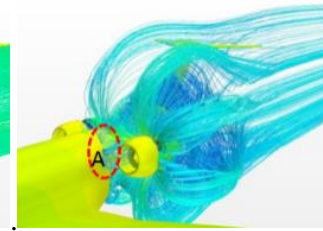

(b) $0.05 \mathrm{Ma}$
Fig. 17. Space streamline distribution of reverse thrust airflow.

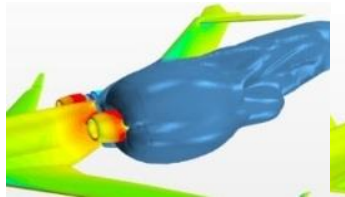

(a) $0.1 \mathrm{Ma}$

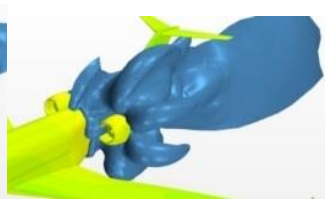

(b) $0.05 \mathrm{Ma}$
Fig. 18. Iso-surface of total temperature at 300K.

Fig. 18 shows the iso-surface of the engine with a total temperature of $300 \mathrm{~K}$ at different running speeds. The influence range of radial and course direction of reverser flow increases and gradually occupies the front side space of the engine lip with speed of airplane skidding decreasing.

By comparing with Fig. 15 and Fig. 17, there is a certain difference between the reverser flow field of a single engine and that of the aircraft/engine integration. The outflow of reverser flow in the wingspan direction cannot be guaranteed to be symmetrical with the obstruction of aircraft fuselage, while in the vertical direction, the reverser flow presents a relatively symmetrical flow field, and the lower side is slightly affected by the ground. By comparing with Fig. 15 (b) and Fig. 17(b) in the same way, it can be clearly seen that when the single engine model has no fuselage, a large vortex appears on the upper and lower sides of the engine and in front of the lip, and the influence range is significantly larger than that of aircraft/engine integrated model. The movement range of reverser airflow in the radial and circumferential direction is significantly greater than in the case of aircraft/engine integration in the absence of airframe interference. However, reverser flow strikes the fuselage surface on the side close to the fuselage and presents a reverse pressure gradient in the situation of the interference of the fuselage of the aircraft. An obvious vortex takes shape near the engine lip under the relative flow blowing, which is shown in region A in Fig. 17(b). In addition, the wing may also interfere with the reverser flow when calculating the aircraft/engine integration model. Reverser flow gradually moves to the space region between the engine lip and wings. The above will interfere with the free incoming flow air drawn in by the engine. 
This suggests that reverser flow is susceptible to be affected by the adjacent fuselage and wing areas, and these structures also limit the development of backward thrust.

In order to further analyze the influence of fuselage interference on the reverse thrust flow, the vorticity distribution and the two-dimensional streamline distribution on the longitudinal section of the engine are compared. Fig. 19 shows the streamline distribution on the longitudinal section of the engine as the incoming flow is $0.1 \mathrm{Ma}$. Fig. 19(a) shows that the vorticity of the outlet area decreases after the reverse thrust flows out, which means the velocity gradient and the momentum loss reduces. Fig. 19(b) shows that the vorticity of the outlet area with the aircraft fuselage interference. Compared to the case of the single engine, the vorticity of the outlet area increases, which means the momentum of reverse thrust flow increases. Therefore, the reverse thrust decreases with the aircraft fuselage interference, the value of reverse thrust decreases by $5 \%$ ((4300$4085) / 4300=5 \%$ ).

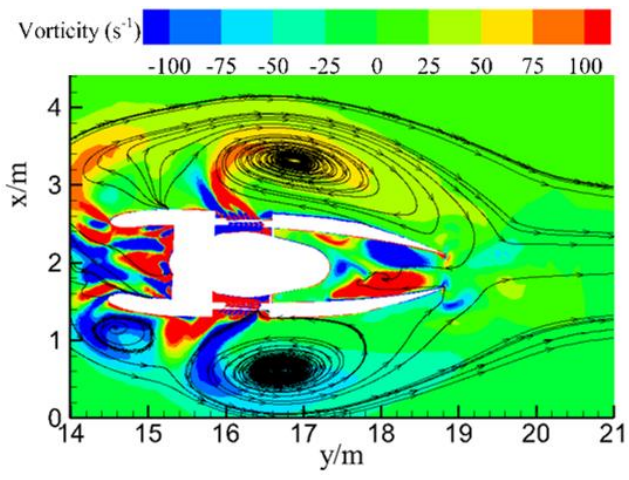

(a) Individual engine model

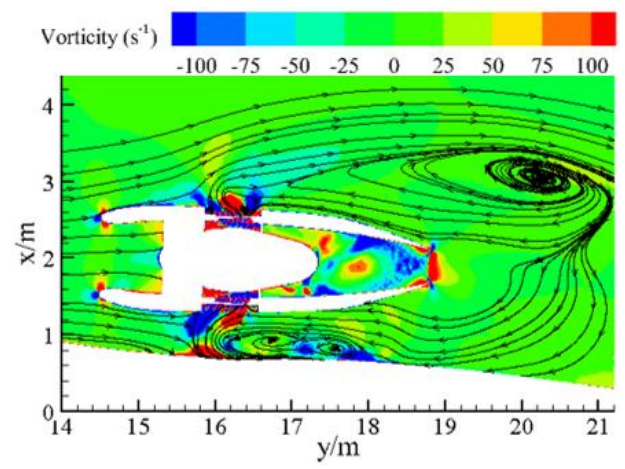

(b) Aircraft/engine integration model

Fig. 19. Streamline distribution on the longitudinal section of the engine (0.1Ma).

Generally speaking, the fuselage and wing of the aircraft restrict the development of the reverser air flow in the tail-lift engine layout to some extent, and there are obvious interference effects. Therefore, the change of engine layout will cause the change of the flow space of reverser thrust airflow, and then affect the streamline distribution of reverser thrust airflow when designing the business aircraft with a tail-lift layout. In this way, it is necessary to explore the influence of the flow field under different engine layouts.

\subsection{Influence of Engine Nacelle Layouts}

The reverse thrust airflow flow field of different engine nacelle layouts is calculated. Fig.20 shows the space streamline distribution of reverse thrust airflow under different nacelle layouts as inlet Mach number is 0.1 . The momentum loss gradually decreases, and the reverse airflow flows to the rear of the engine, and then changes. The reverse thrust airflow does not flow to the nearby of the inlet, and then it goes to the rear of the engine. Fig. 20(b) shows the pressure cloud and streamline of basic engine layout, the layout is defined the engine reference position. It is obvious that the reverser airflow flows towards the engine lip direction, and then changes to the exhaust direction, the surface pressure of the adjacent fuselage and pod is relatively larger. After nacelle moves towards the exhaust direction, the distance between nacelle and tail reduces, as the Fig. 20 (a) shows that the movement ranges of streamline becomes smaller at the radial direction clearly.

As the engine nacelle moves forward, the fuselage coarsens and the distance between wing and engine nacelle reduces, which makes the space between wing and fuselage smaller. As a result, the reverse airflow flow resistance increases, and the energy loss of the reverse thrust flow increases, so that the momentum of reverse airflow reduces gradually. As the Fig. 20(c) and Fig. 20(d), the streamline is overall similar, a part of streamline flows to the engine inlet nearby, the forward movement distance of the reverse thrust flow is relatively increased, which may also produce better reverse thrust effect. It is obvious that the turning point of the reverse flow direction is closer to the engine inlet when the engine nacelle moves forward by $22 \% L$.

Fig. 20(e) shows the space streamline distribution of reverse thrust airflow when the nacelle moves laterally. The wing of plane is a swept-back wing, the average distance between nacelle and wing reduces, which also affect the reverse airflow developments, as the Fig. 20(e) shows that the movement range of reverse airflow does not enlarge and reduces at the lateral direction. Fig. 20(f) and Fig. 20(g) shows the space streamline distribution of reverse thrust airflow when nacelle moves upward and downward. Compared with the streamline of nacelle moving downward, the radial movement range of reverse airflow is relatively larger when nacelle moves upward. When engine nacelle moves upward, the vertical distance increases between engine and wing. It's the opposite when the engine nacelle moves vertical downward. The motion space of air flow over the wing increases when engine nacelle moves upward, and the momentum loss of the income flow decreases relatively, the resistance towards the reverse airflow enhances, but the fuselage resistance to reverse airflow reduces. And the upper region of fuselage is relatively smaller than belly, so the fuselage effect on the reverse airflow is bigger when the nacelle moves down. When the engine nacelle moves downward, the vertical distance between the nacelle and wing decreases and the distance between the nacelle and the belly of the aircraft decreases, which causes that the flow space 


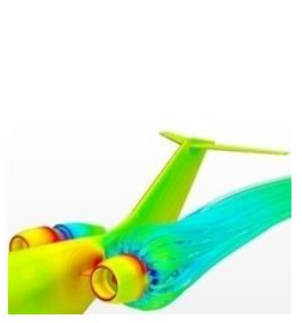

(a) forward $-1.15 \% \mathrm{~L}(\mathrm{i}=1)$

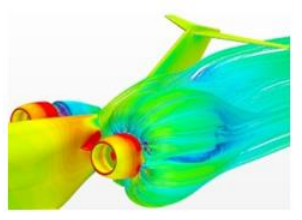

(e) lateral $1.15 \% \mathrm{~L}(\mathrm{i}=5)$

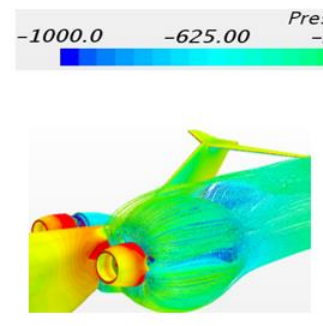

(b) reference position $(\mathrm{i}=2)$

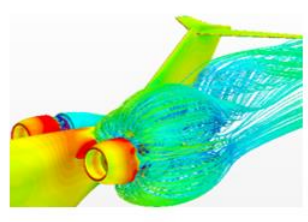

(f) upward $3.5 \% \mathrm{~L}(\mathrm{i}=6)$

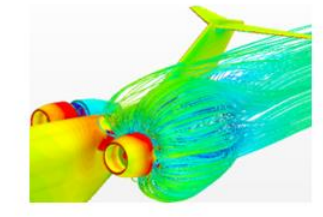

(c) forward $11 \% \mathrm{~L}(\mathrm{i}=3)$

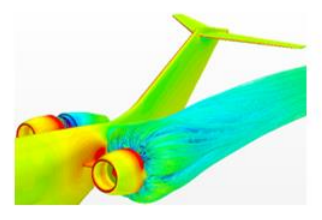

(g) downward $3.5 \% \mathrm{~L}(\mathrm{i}=7)$

Fig. 20. Space streamline distribution of reverse thrust airflow.

of the incoming flow flowing through the wing increases slightly, so that the attenuation of the incoming flow decreases, the flow resistance of the reverse flow increases, and the influence range of the reverse thrust flow is small.

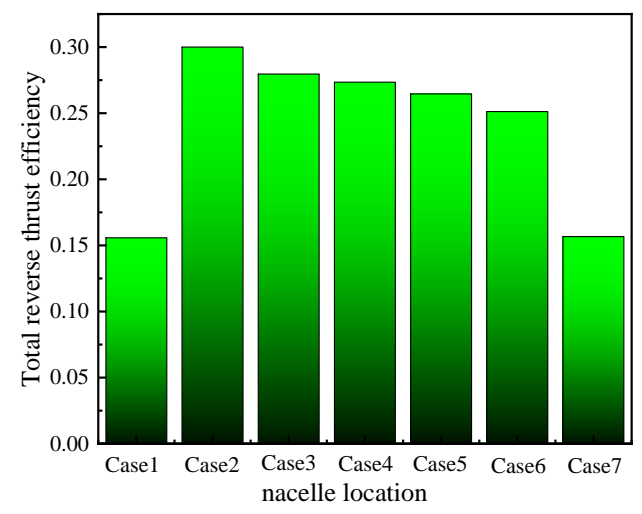

Fig. 21. Total reverse thrust efficiency of different nacelle layouts.

Fig. 21 shows total reverse thrust efficiency $\eta_{\text {rev }}$ of different engine nacelle layouts. When the nacelle moves forward by $-1.15 \% \mathrm{~L}$ (case 1 ), the total reverse thrust efficiency $\eta_{\text {rev }}$ of basic layout (case2) reduces, and as the nacelle moves forward by $11 \% \mathrm{~L}$ (case 3 ) and $22 \% \mathrm{~L}$ (case 4$), \eta_{\text {rev }}$ increases compared to the case1. So as the nacelle moves forward by $-1.15 \% \mathrm{~L}, 11 \% \mathrm{~L}$ and $22 \% \mathrm{~L}$, the total reverse thrust efficiency $\eta_{\text {rev }}$ begin reduces and then increases gradually. Fig. 22 shows total pressure recovery coefficient ratio $\sigma$ of different nacelle layouts. When the nacelle moves forward, $\sigma$ begins to reduce and then increases gradually, the numerical changing trend is same to the $\eta_{\text {rev }}$. As engine nacelle moves forward, the space between nacelle and wing reduces, the resistance of reverse airflow increases, and when the nacelle moves forward continually, the influence of tail reduces. So the corresponding reverse airflow flow loss presents a tendency to decrease and then increases. And when the nacelle moves upward, downward and moves lateral, especially moves back, the $\sigma$ reduces in contrast to the reference layout, and the $\eta_{\text {rev }}$ also decreases.

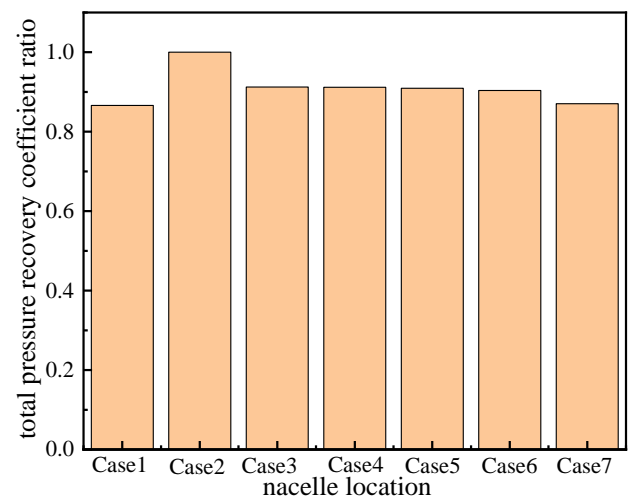

Fig. 22. Total pressure recovery coefficient ratio of different nacelle layouts.

The total temperature of the air flow increases after the engine air flow passes through the friction of the inlet and the fan compression does work. The total temperature decreases after the reverse thrust airflow mixing with the main flow, however, the total temperature of the reverse thrust flow is still higher than that of the ambient flow.

Fig. 23 shows the iso-surface of the total temperature at $300 \mathrm{~K}$ when the inlet Mach number is 0.1 at different nacelle layouts. In Fig. 20, the airflow ejected from the thrust device is not re-inhaled, and there is no obvious re-inhalation phenomenon. As can be clearly seen from Fig. 23(c), the range of motion of the reverse thrust airflow increases, and some of the reverse thrust airflow flows to the nearby of the engine inlet. Fig. 23(f) and Fig. 23(g) show that compared to the model of engine nacelle moves downward, the flow space of the reverse thrust airflow increases relatively when engine nacelle moves upward, and its forward flow distance and influence range also increase correspondingly. 


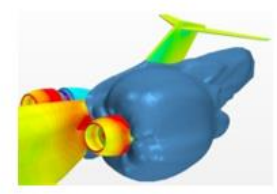

(a) forward $-1.15 \% \mathrm{~L}(\mathrm{i}=1)$

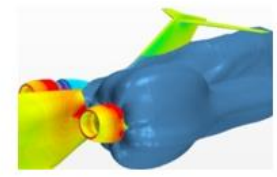

(e) lateral $1.15 \% \mathrm{~L}(\mathrm{i}=5)$

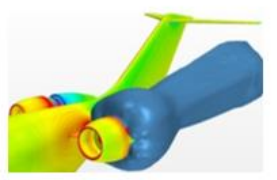

(b) basal position $(\mathrm{i}=2)$
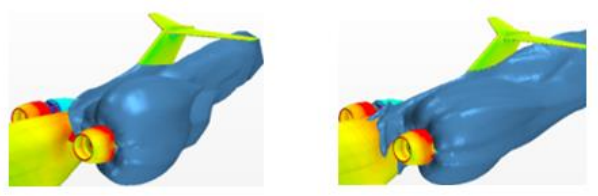

(c) forward $11 \% \mathrm{~L}(\mathrm{i}=3)$

(d) forward $22 \% \mathrm{~L}(\mathrm{i}=4)$

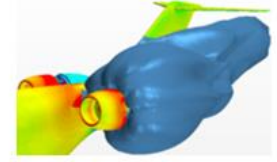

(f) upward $3.5 \% \mathrm{~L}(\mathrm{i}=6)$

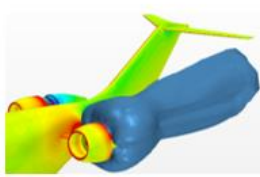

(g) downward $3.5 \% \mathrm{~L}(\mathrm{i}=7)$

Fig. 23. Iso-surface of the total temperature at $300 \mathrm{~K}$.
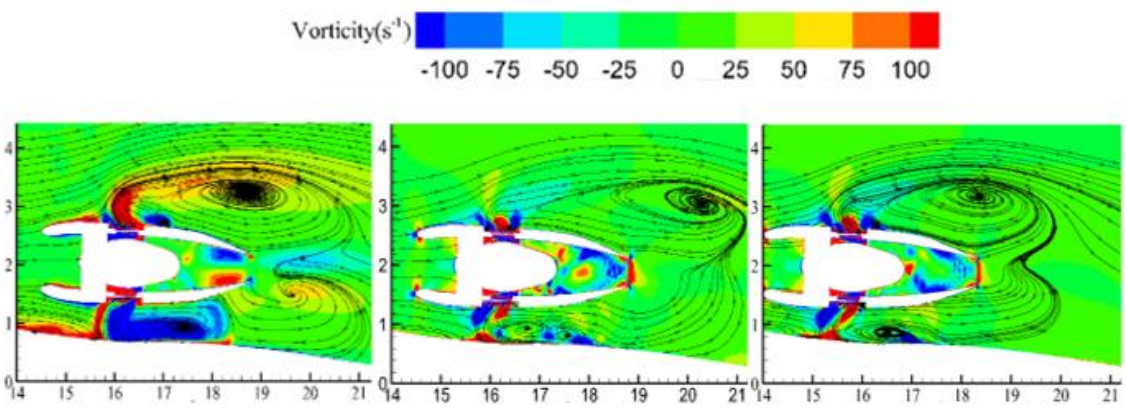

(a) case 1

(b) case 2

(c) case 3

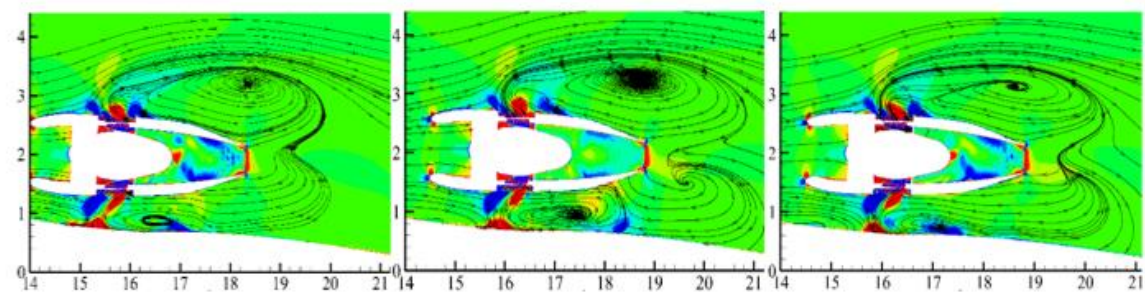

(d) case 4

(e) case 5

(f) case6

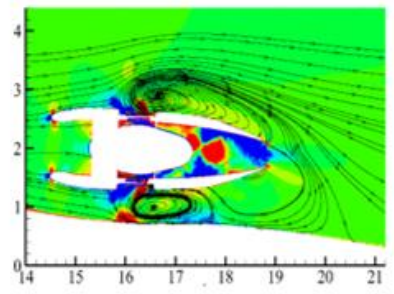

(g) case7

Fig. 24. Streamline distribution on the longitudinal section of the engine.

In order to further analyze the influence of different nacelle positions on the thrust flow, the vorticity and streamline distribution on the longitudinal section of engine are compared and analyzed. Fig. 24(a) and Fig. 24(b) show that compared with the case of basal position, the vorticity of the longitudinal section increases as the engine move backward. In addition, the size of the reflux vortex increases after the reverse thrust airflow flows out. So the reverse thrust decreases by $48 \%$. When the engine nacelle moves forward, the space of reverse thrust airflow flowing decreases. As Fig. 24(c) and Fig. 24(d) show that the vorticity of longitudinal section increases, thus the reverse thrust deceases.

Fig. 24(e) shows that the vorticity of longitudinal when the engine nacelle moves toward latera, the distance between engine nacelle and fuselage 
increases, so that the space in which reverse thrust airflow flowing out increases. The vorticity of the longitudinal section does not change almost. However, the size of the reflux vortex increases and the flow loss further increases, thus the reverse thrust decreases.

Fig. 24(f) and Fig. 24(g) show that the vorticity of longitudinal section as engine nacelle moves upward and downward. When the engine nacelle moves upward and downward, the vorticity longitudinal section do not change almost. But the size of the reflux vortex increases, especially when the engine nacelle moves downward. Under the condition of the same vorticity, the large size of the vortex indicates that the flow loss is large. Therefore, compared to the case of engine moves upward, the flow loss enlarges and the reverse thrust decreases when engine nacelle moves downward

\section{CONCLUSION}

In this paper, the full 3D numerical simulation of turbulence flow field of a turbofan engine with cascade type thrust reversal device is carried out. Firstly, the aerodynamic performance of aircraft propulsion system integration on different engine nacelle layouts is compared and analyzed. Then, the effect of fuselage on reverse thrust flow characteristics is studied. At last, the reverse thrust flow characteristics of different engine nacelle layouts are investigated. The specific conclusions are as follows:

(1) When the engine nacelle moves forward horizontally by $11 \% L$ and $22 \% L$, the surface pressure coefficient on the upper side of wing increases obviously from leading edge of wing to 0.4 chord, and the corresponding lift coefficient of the wing decreases $21.2 \%$ and $45.02 \%$ respectively. The lift coefficient of the wing decreases $2.4 \%$ and $4.82 \%$ respectively as the engine moves subsidence by $-3.5 \% L$ and $3.5 \% \mathrm{~L}$.

(2) Numerical simulation results of single engine and aircraft/engine integrated reverse flow field show that the area affected by the reverse thrust flow varies in size at the same incoming velocity. The reverse thrust airflow forms a vortex region near the engine lip as the incoming velocity is $0.05 \mathrm{Ma}$. Both the single engine and aircraft/engine integrated two cases occur the phenomenon of re-inhalation may occur.

(3) There are some differences in the reverser flow field between single engine and aircraft/engine integrated. The movement range of reverser airflow in the radial and circumferential direction without airframe interference is significantly greater than in the case of aircraft/engine integration. The reverser flow in the direction of the wing spread is not symmetrical at any glide speed when obstructed by the fuselage in the presence of airframe interference. The reverser flow is susceptible to be interfered by the adjacent fuselage and wing sections, and the development of reverse thrust flow is limited.

(4) Compared to the result of the basal nacelle location, when the engine nacelle moves forward, the $\eta_{\text {rev }}$ begins to reduce gradually, the $\sigma$ keep the same trend; as the nacelle moves backward, upward and moves lateral, the $\sigma$ reduces and the $\eta_{\text {rev }}$ also decreases. The reverse thrust performs badly as engine nacelle moves backward and lateral horizontally.

\section{ACKNOWLEDGMENTS}

The authors gratefully acknowledge the financial supports for the project from the National Science and Technology Major Project of China (2017-III0010-0036). In addition, Xingsi Han acknowledges the support of the Jiangsu Specially Appointed Professor Program.

\section{REFERENCE}

Andreopoulos, J. and W. Rodi (1984). Experiment investigation of jets in a crossflow. Journal of Fluids Engineering 138, 93-127.

Asbury, S. C. and A. J. Yetter (2000). Static Performance of Six Innovative Thrust Reverser Concepts for Subsonic Transport Applications. NASA TM-2000-210300.

Chen, C. (2001). Computational procedures for complex three-dimensional geometries including thrust reverser effluxes and APUs. AIAA 2001-3747.

Chen, G. and R. Hu (2017). Analysis and validation of thrust-reversers aerodynamic characteristics for civil aircraft. Aeroengine 4(02), 56-61.

Chen, Z. (2014). Research on the structure and aerodynamic performance of the cascade thrust reverser. Nanjing University of Aeronautics and Astronautics, 2014.

Chen, Z., Y. Shan, X. G. Shen, J. Z. Zhang and W. R. Shao (2016). Numerical simulation on reingestion characteristics under landing state for thrust reverser. Journal of Aerospace Power 31(03), 733-739.

Dang, Y., K. Liu, Z. Tan and X. Jiang (2018). Numerical study of engine installed effect on a tail-mounted civil aircraft thrust characteristic. Journal of propulsion technology 39(8), 17121719.

De Andrade, F. O., S. B. Ferreira and L. F. F. da Silva (2006). Study of the influence of aircraft geometry on the computed flow field during thrust reversers operation. AIAA 2006-3673.

Du, G. and J. Jin (2007). Large transport aircraft engine thrust counter device, Large aircraft key technology high-level BBS and 2007 annual conference proceedings of CSAA: power topic 21. Shenzhen: CSAA 2007, 1-11. 
Duan, Z., Z. Liao and R. Qian (2016). Civil aircraft thrust reverser cascade configuration and airplane integration evaluation through CFD. Physics of Gases 1(06), 49-55.

Fang, C. (2008). Key technologies for high bypass turbofan engines. International Aviation (1), $38-40$.

He, Y. and Y. Liu (2010a). Effect of blade geometric intake angle and pressure ratio on cascade thrust reverser performance. Science Technology and Engineering 10(02), 458-465.

He, Y. and Y. Liu (2010b). Effect of blade solidity and pressure ratio on cascade thrust reverser performance. Aeroengine 36(02), 16-21.

He, Y. and Y. Liu (2012). Effect of blade exit angle and pressure ratio on cascade thrust reverser performance. Science Technology and Engineering 12(01), 89-96.

Jin, B., W. Xing and D. Liu (2004). Thrust Reversers of Aircraft/engine Propulsion System. Aeroengine 30(4), 48-52.

Levy, D. W., J. V. Zickuhrt, J. Vassberg, S. Agrawal, R. A. Wahls, S. Pirzadeh and M. J. Hemsch (2002). Summary of data from the first AIAA CFD drag prediction workshop, AIAA-20020481.

Li, D. X., K. L. Zhao, Q. Z. Wang, M. J. Sheng, Y. Huang and M. H. Zhang (2012). Wind tunnel test for turbofan engine re-ingestion characteristics of civil aircraft. Journal of Experiments in Fluid Mechanics, 26(05), 31-35.

Qian, R., Z. Zhu and Z. Duan (2011). Thrust Reverser Optimization for Safety with CFD. Procedia Engineering 17, 595-602.

Rao, Q. (2014). Research on engine thrust reverser. Science Mosaic 02.015,91-94.

Rossow, C. C., J. L. Godard, H. Hoheisel and V. Schmitt (1994). Investigations of propulsion integration interference effects on a transport aircraft configuration. Journal of Aircraft 31(5), 1022-1030.

Sha, J. and J. Xu (2007). Research progress of engine thrust reverser device //Large aircraft key technology high-level BBS and 2007 annual conference proceedings of CSAA: power topic 22. Shenzhen: CSAA, 2007:1-6.

Shan, Y., X. Shen, J. Zhang, S. Shang and W. Shao (2010). Numerical study for the effects of cascade geometry parameters on cascade-type thrust reverser performances. Journal of civil aviation university of China 28(3), 10-14.

$\mathrm{Su}$, L. and M. Mungal (2004). Simulation measurements of scalar and velocity field evolution in turbulent crossflowing jets. Journal of Fluid Mechanics 513, 1-45.

Trapp, L. G. and G. L. Oliveira (2003). Aircraft thrust reverser cascade configuration evaluation through CFD. AIAA 2003-723.

Wang, D. and Z. Y. Zhang, S. C. Hao, D. Wang and Z. Zhao (2018). Flow simulation and experiment of gradual reverse thrust plane cascade endwall. Aeroengine 44(1) 63-68.

Wang, P., Y. C. Cui, Y. C. Chen, X. D. Zhang, Z. Deng, S. P. Lu and J. F. Teng (2019). Experimental and Numerical Research on the Aerodynamic Performance of Wide-Body Aircrafts Thrust Reverser Cascade. Journal of Shanghai Jiao Tong University 53(4), 413-422.

Wang, Z., X. Shen and J. Hu (2017). Prediction and evaluation of aerodynamic stability of high bypass ratio turbofan engine deployed with thrust reverser. Acta Aeronautica et Astronautica Sinica 38(02), 120192.

Wang, Z., X. Shen, G. X. Hujun and L. Liu (2016). Numerical investigation on influence of reverser flow to engine inlet flow field. Journal of Aerospace Power 31(04), 918-926.

Yetter, J. A. (1995). Why do airlines want and use thrust reversers. NASA TM-109158.

Yi, X., K. C. Wang, H. L. Ma and G. L. Zhu (2014). Analysis of aerodynamic characteristics of engine thrust reverser in civil aircraft. Applied Mathematics and Mechanics 35(S1), 85-89.

Zhang, G. D. and Q. Wang (2010). The influence of cascade solidity and flow angle of inlet and outlet on aerodynamic performance of cascade thrust reverser. Aircraft Design 30(4), 51-56.

Zhao, H. (2019). Numerical simulation study on influence of reverser flow on high bypass ratio turbofan engine inlet flow field. Gas Turbine Experiment and Research 32(6), 20-25.

Zuo, Z. and R. Qian (2007). Numerical simulation investigation of effect of thrust reverser operating on engine inlet flow distortion for civilian transport. Aircraft Design 27(05), 6064. 